\title{
Influence of baseline severity on antidepressant efficacy for anxiety disorders: meta-analysis and meta-regression
}

Ymkje Anna de Vries, Peter de Jonge, Edwin van den Heuvel, Erick H. Turner and Annelieke M. Roest

\section{Background}

Antidepressants are established first-line treatments for anxiety disorders, but it is not clear whether they are equally effective across the severity range.

\section{Aims}

To examine the influence of baseline severity of anxiety on antidepressant efficacy for generalised anxiety disorder (GAD), social anxiety disorder (SAD), obsessive-compulsive disorder (OCD), post-traumatic stress disorder (PTSD) and panic disorder

\section{Method}

Fifty-six trials of second-generation antidepressants for the short-term treatment of an anxiety disorder were included. Baseline and change scores were extracted for placebo and treatment groups in each trial. Mixed effects meta-regression was used to investigate the effects of treatment group, baseline severity and their interaction.

\section{Results}

Increased baseline severity did not predict greater improvement in drug groups compared with placebo groups.
Standardised regression coefficients of the interaction term between baseline severity and treatment group were 0.04 $(95 \% \mathrm{Cl}-0.13$ to $0.20, P=0.65)$ for $\mathrm{GAD},-0.06(95 \% \mathrm{Cl}$ -0.20 to $0.09, P=0.43)$ for SAD, $0.04(95 \% \mathrm{Cl}-0.07$ to 0.16 , $P=0.46)$ for $O C D, 0.16(95 \% \mathrm{Cl}-0.22$ to $0.53, P=0.37)$ for PTSD and $0.002(95 \% \mathrm{Cl}-0.10$ to $0.10, P=0.96)$ for panic disorder. For OCD, baseline severity did predict improvement in both placebo and drug groups equally $(\beta=0.11,95 \% \mathrm{Cl}$ 0.05 to $0.17, P=0.001$ ).

\section{Conclusions}

No relationship between baseline severity and drug-placebo difference was found for anxiety disorders. These results suggest that if the efficacy of antidepressants is considered clinically relevant, they may be prescribed to patients with anxiety regardless of symptom severity.

\section{Declaration of interest \\ None.}

\section{Copyright and usage}

(c) The Royal College of Psychiatrists 2016.
Anxiety disorders are the most common mental disorders, with a combined 12-month prevalence of $18.1 \%$ and a lifetime prevalence of $28.8 \%$ in the USA. ${ }^{1,2}$ Owing to their high prevalence, combined with an often early onset and chronic course, ${ }^{2-4}$ anxiety disorders are the second most important cause of disability worldwide within the group of mental and behavioural disorders. ${ }^{5}$ Antidepressants, including the secondgeneration selective serotonin reuptake inhibitors (SSRIs) and serotonin-noradrenaline reuptake inhibitors (SNRIs), have been found to be efficacious in the treatment of most anxiety disorders, including generalised anxiety disorder (GAD), social anxiety disorder (SAD), obsessive-compulsive disorder (OCD), posttraumatic stress disorder (PTSD) and panic disorder. ${ }^{6-10}$ However, research in major depressive disorder has suggested that the efficacy of antidepressants depends on the baseline severity of depression. One meta-analysis of the Food and Drug Administration (FDA) database of randomised controlled trials (RCTs) of second-generation antidepressants showed that trials with higher mean baseline severity scores were more likely to be positive, ${ }^{11}$ and another found a significant interaction between baseline severity and treatment group in predicting improvement, such that the drug-placebo difference is relatively small at low levels of initial severity. ${ }^{12}$ Similarly, an additional meta-analysis found no statistically significant difference between antidepressants and placebo in people with subthreshold (minor) depression. ${ }^{13}$ Of four analyses using individual patient data, three found that baseline severity of depression was associated with antidepressant efficacy, ${ }^{14-16}$ whereas another large study did not find a significant association. ${ }^{17}$

Much less is known about the relationship between baseline severity and antidepressant efficacy in the context of anxiety disorders. For OCD, a meta-analysis of 24 antidepressant RCTs found that baseline severity predicted greater improvement in both placebo and drug groups, but there was no evidence of an interaction between baseline severity and treatment group. ${ }^{18}$ Recently a meta-analysis of 12 RCTs of paroxetine for GAD and panic disorder found no evidence of an interaction effect. ${ }^{19}$ However, pooling trials for different disorders may obscure differences between disorders; it also necessitated the use of a secondary outcome for panic disorder, rather than the primary, panic-specific outcome in these trials. Other single trials and small pooled analyses that have examined this question have reached contradictory conclusions. ${ }^{20-22}$ The evidence to date, therefore, is (perhaps with the exception of OCD) conflicting, minimal or absent altogether. To our knowledge, no study has comprehensively investigated whether baseline severity predicts antidepressant efficacy in all anxiety disorders. If antidepressant efficacy does depend on baseline anxiety severity, this has important consequences for the continued development of guidelines for the treatment of these disorders. We therefore conducted a meta-analysis and meta-regression of RCTs of SSRIs and SNRIs for the short-term treatment of anxiety disorders using trials submitted to the FDA.

\section{Method}

We obtained drug approval packages (reviews) for all SSRIs and SNRIs approved by the FDA for the short-term treatment of five anxiety disorders: GAD, SAD, OCD, PTSD and panic disorder. ${ }^{23}$ Reviews were downloaded from the FDA website when available or requested from the FDA's Division of Freedom of 
Information. ${ }^{24}$ From the FDA reviews we extracted data on the duration of the trial, drug dose, number of participants, mean score on the primary outcome measure at baseline and end-point (with standard error (s.e.) or standard deviation (s.d.) if available), and the mean change in the primary outcome measure (with s.e. or s.d. if available), for drug and placebo groups separately. For GAD the primary outcome measure in all included trials was the change from baseline on the Hamilton Rating Scale for Anxiety (HRSA); for SAD it was the change on the Liebowitz Social Anxiety Scale (LSAS); for OCD, the change on the Yale-Brown Obsessive Compulsive Scale (Y-BOCS); and for PTSD, the change on the Clinician-Administered PTSD Scale, part 2 (CAPS-2). For panic disorder the primary outcome for most trials was the number of panic attacks over a period of $1-3$ weeks, dichotomised into 0 (remitted) or $\geqslant 1$ (not remitted); four trials, however, used the change in number of panic attacks as a primary outcome measure. Therefore, for this disorder we also extracted data on the proportion of participants who were free from panic attacks at end-point (remission rate). Furthermore, panic disorder trials reported a baseline score as the number of panic attacks in the 1,2 or 3 weeks before baseline; we converted all scores to a 2 -week time frame.

Data were extracted from the (modified) intention-to-treat analyses only, which used the last observation carried forward (LOCF) method to handle missing data from those leaving the study. Data were extracted preferably from the statistical review; however, we gave preference to other documents within the drug approval package (e.g. the medical review or administrative correspondence) if they provided more complete data (e.g. the s.e. or s.d.) as well as the mean change. If complete data were not available in the FDA review we attempted to obtain the missing data from secondary sources (trial registries and published journal articles).

\section{Outliers and missing data}

Exploration of mean baseline scores, stratified by disorder, revealed no outlier in the data-sets for GAD, SAD, OCD and PTSD. For panic disorder, however, one placebo group and one drug group (from two separate trials) had mean baseline scores more than 2 s.d. greater than the overall mean baseline score (after log-transformation to normalise the distribution); we therefore excluded these groups (but not other groups within the same trial) from our analysis. We were able to obtain data on the mean change score and its s.e. or s.d. (or the remission rate for panic disorder) for 46 out of 56 trials. For 40 trials the FDA review provided complete data; for 2 trials we obtained data on the s.e./s.d. from the GlaxoSmithKline trial registry and for 4 trials we obtained data on the s.e./s.d. ( 3 trials) or the remission rate (1 trial) from the matching published journal articles, ${ }^{23}$ after verifying that mean baseline and change scores matched those in the corresponding FDA review. For 10 out of 56 trials not all required data could be obtained from any source. Information on the s.e./s.d. of the change score was missing for 1 PTSD and 6 OCD trials, whereas information on the remission rate was missing for 3 panic disorder trials. For all 10 trials, however, information on the mean change score was available. For PTSD and OCD the change score itself was strongly correlated with its s.d. in groups without missing data; we therefore imputed these missing s.d.s based on the change score, group membership and their interaction. For panic disorder, end-point score was strongly correlated with the remission rate, and we therefore imputed missing remission rates based on end-point score, group membership and their interaction. Imputation was performed separately per disorder in SPSS 20, using multiple imputation with fully conditional specification based on linear regression in order to create ten imputed data-sets.

\section{Statistical analysis}

For our main analysis we calculated effect sizes separately for each treatment group, as a single-group pre-post effect size. This approach allowed us to investigate not only the relationship between baseline severity and antidepressant efficacy (drugplacebo difference) but also its underlying cause (i.e. change in the placebo response $v$. change in the drug response). For GAD, SAD, OCD and PTSD, the standardised mean difference (SMD) was first calculated based on the (within-group) change score and its standard deviation according to the formula $S M D=D /$ $S D_{D}$, where $D$ signifies the change score and $S D_{D}$ the standard deviation of the change score. ${ }^{25}$ By using this formula we assume that the correlation between baseline and end-point scores is 0.5 , as the true correlation is unknown. We then applied Hedges' correction for small sample size, where $n$ indicates the number of participants in a group: ${ }^{25}$

$$
g=\left(1-\frac{3}{4(n-1)-1}\right) \times S M D
$$

The standard error of Hedges' $g$ was computed as follows: ${ }^{25}$

$$
S E=\left(\frac{1}{n}\right)\left(\frac{n-1}{n-3}\right)\left(1+n \times g^{2}\right)-\left(g^{2} /\left(1-\frac{3}{4(n-1)-1}\right)^{2}\right)
$$

For panic disorder we selected the remission rate itself as our effect measure. To obtain a single effect size for the antidepressant arms of fixed-dose studies, we used a fixed effects, inverse variance-weighted model to pool these drug groups into one estimate of effect size with its standard error for GAD, SAD, OCD and PTSD. A pooled remission rate was derived for panic disorder by calculating the sample size-weighted average of the remission rates in the different dose groups. For all disorders a pooled baseline score was derived by calculating the sample sizeweighted mean of the baseline scores in the different dose groups. Pooling different dose groups may not be appropriate in the presence of a dose-response relationship, but with the exception of venlafaxine such relationships usually cannot be demonstrated with second-generation antidepressants. ${ }^{26}$

All analyses were performed in Stata 13. We performed metaanalyses using the metan command, applying a random effects (DerSimonian-Laird) model to obtain summary statistics by disorder and group. To measure heterogeneity, the heterogi module within Stata was used to calculate $I^{2}$ and its $95 \%$ confidence interval. ${ }^{27}$ Meta-regression was then performed separately per disorder using the metareg command. All metaregressions were based on a mixed effects model, used restricted maximum likelihood (REML) estimation of the residual between-study variance, and included group, baseline severity and their interaction as predictors. The dependent variables were Hedges' $g$ for GAD, SAD, OCD and PTSD and remission rate for panic disorder. Studies were weighted according to the inverse of their variance. For OCD, PTSD and panic disorder, metaregression estimates from the ten multiply imputed data-sets were combined using the $m i$ suite of commands in Stata.

\section{Sensitivity analyses}

As a secondary analysis we calculated Hedges' $g$ for the drugplacebo difference directly from the exact $P$-value for the statistical test performed at end-point (or alternative methods as required; 
see Roest et al). ${ }^{23}$ The trial baseline severity score was calculated as the sample size-weighted average of all groups (drug as well as placebo) included in the trial. We performed meta-regressions separately for each disorder using the metareg command in Stata. The dependent variable was the drug-placebo difference (Hedges' $g$ ) for all disorders, and baseline severity was the only predictor in this analysis. Additionally, to increase statistical power and improve generalisability of results, we expanded our data-set by including extra trials. We included active comparator arms and trials that were not conducted for the purpose of marketing approval, such as trials of other medications (e.g. antipsychotics) in which the antidepressant was used as an active comparator. Trials were obtained from the most recent meta-analyses examining (pharmacological) treatment of anxiety disorders. ${ }^{8,28,29}$ As this introduced trials with small sample sizes, additional heterogeneity and likely reporting bias, this expanded set of trials was examined as a secondary analysis only. We included parallelgroup, placebo-controlled trials with a similar duration (8-16 weeks) to our primary set. Trials with a sample that partially or fully overlapped with a trial in the primary set were excluded, as were trials that did not use a compatible outcome (e.g. HRSA, LSAS, Y-BOCS, CAPS or number of panic attacks as a primary or secondary outcome). We repeated our primary analysis for this expanded set of trials.

\section{Results}

A total of 21 reviews were obtained, concerning nine formulations of SSRIs and SNRIs: escitalopram, duloxetine, fluoxetine, fluvoxamine, fluvoxamine controlled release (CR), paroxetine, paroxetine $\mathrm{CR}$, sertraline and venlafaxine extended release (XR). These reviews comprised 59 RCTs: 11 for GAD, 11 for SAD, 13 for OCD, 7 for PTSD and 17 for panic disorder (see online Table DS1). However, we excluded 2 trials for SAD and 1 trial for panic disorder (Table DS1) because baseline severity scores were not comparable with the other trials. Specifically, the excluded SAD trials did not use the LSAS and the excluded panic disorder trial that failed to distinguish between full and limited-symptom panic attacks. Consequently, 56 trials were included in this study. We used only data concerning doses recommended by the FDA; therefore, for paroxetine we excluded the $20 \mathrm{mg}$ dose in one OCD trial and the $10 \mathrm{mg}$ and $20 \mathrm{mg}$ doses in one panic disorder trial, as only doses of $40 \mathrm{mg}$ or higher were judged to be effective for these disorders.

All included trials were short-term, randomised, double-blind and placebo controlled. Some trials also used active comparators, but data from these were not included in our primary analyses. Trial duration was 12 weeks for all SAD and PTSD trials, 8-10 weeks for GAD trials, 8-16 weeks for OCD trials and 10-12 weeks for panic disorder trials. Trials included adults of 18 years or older (with the exception of 5 OCD trials that included adolescents together with adults) who met DSM-III-R or DSM-IV criteria for the anxiety disorder under investigation. The majority of trials (71\%) used a flexible dose design, allowing investigators to titrate the dose according to the patient's response; the remainder (29\%) used a fixed-dose design with patients randomised to one of two or three different dosage groups.

\section{Participants and baseline severity}

The 56 trials included 55 placebo groups and 78 drug groups, which were pooled into 56 drug groups. The total number of participants was 14710 , of whom 6386 were randomised to receive placebo and 8324 were randomised to the drug groups. Baseline severity was generally in the moderate to severe range. Participant numbers and baseline severity scores are shown in online Table DS2. A complete list of included trials, with baseline and change scores, is provided in online Table DS3.

\section{Meta-analysis}

We performed a meta-analysis of effect sizes, stratified by disorder and group (Table 1). The placebo group effect size was smallest for OCD (0.49), followed by SAD (0.65). These placebo effects were $59 \%$ and $60 \%$ respectively of the effects found for the corresponding drug groups. In contrast, for GAD and PTSD the placebo effect sizes were 1.03 and 0.97 respectively; these effects were $76 \%$ and $83 \%$ respectively of the effects found for the corresponding drug groups. For panic disorder the placebo remission rate was $45 \%$, which was $76 \%$ of the rate found in the drug group. Substantial heterogeneity was present for nearly all groups, with $I^{2}$ ranging from $46 \%$ for the placebo groups in SAD trials to $89 \%$ for the drug groups in PTSD trials, although confidence intervals were generally wide.

\section{Meta-regression}

For both GAD and SAD neither baseline severity nor the interaction between group and baseline severity was a statistically significant predictor of the effect size, although group membership was $(P=0.001$ and $P<0.001$ respectively) (Table 2 and Fig. 1). For OCD the interaction between baseline severity and group membership was not significant, but the main effects of both group membership and baseline severity were (group $P<0.001$; baseline $P=0.001$ ), indicating that the (positive) slope of the association between baseline severity and effect size was similar in the placebo and drug groups. For PTSD none of the predictors achieved statistical significance. For panic disorder we modelled the relationship between baseline severity and the remission rate; in this model the interaction between group membership and baseline severity was not significant (Table 2

Table 1 Meta-analysis of effect sizes

\begin{tabular}{|c|c|c|c|c|c|}
\hline \multirow[b]{2}{*}{ Disorder } & \multicolumn{2}{|c|}{ Placebo group } & \multicolumn{2}{|c|}{ Drug group } & \multirow[b]{2}{*}{ Difference $(95 \% \mathrm{Cl})$} \\
\hline & Effect size $(95 \% \mathrm{Cl})^{\mathrm{a}}$ & $I^{2}(95 \% \mathrm{Cl})$ & Effect size $(95 \% \mathrm{Cl})^{\mathrm{a}}$ & $I^{2}(95 \% \mathrm{Cl})$ & \\
\hline GAD & $g=1.03(0.93$ to 1.13$)$ & 59 (0 to 77 ) & $g=1.35(1.24$ to 1.46$)$ & 72 (41 to 83) & $g=0.32(0.16$ to 0.47$)$ \\
\hline SAD & $g=0.65(0.57$ to 0.74$)$ & 46 (0 to 73 ) & $g=1.08(0.99$ to 1.18$)$ & 51 (0 to 75) & $g=0.43(0.29$ to 0.57$)$ \\
\hline OCD & $g=0.49(0.39$ to 0.59$)$ & $51(1 \text { to } 73)^{b}$ & $g=0.83(0.73$ to 0.92$)$ & $47(0 \text { to } 70)^{b}$ & $g=0.34(0.19$ to 0.48$)$ \\
\hline PTSD & $g=0.97$ (0.81 to 1.13$)$ & $69(11 \text { to } 84)^{b}$ & $g=1.17(0.91$ to 1.44$)$ & $89(80 \text { to } 9)^{b}$ & $g=0.20(-0.15$ to 0.55$)$ \\
\hline Panic disorder & $\mathrm{RR}=0.45$ (0.38 to 0.52) & $80(65 \text { to } 86)^{b}$ & $\mathrm{RR}=0.59$ (0.55 to 0.64) & $72(50 \text { to } 82)^{b}$ & $\mathrm{RR}=0.14$ (0.06 to 0.22$)$ \\
\hline
\end{tabular}




\begin{tabular}{|c|c|c|c|c|}
\hline \multirow[b]{2}{*}{ Predictor $^{a}$} & \multicolumn{2}{|c|}{ Model 1 (with interaction) } & \multicolumn{2}{|c|}{ Model 2 (without interaction) } \\
\hline & $\beta(95 \% \mathrm{Cl})$ & $P$ & $\beta(95 \% \mathrm{Cl})$ & $P$ \\
\hline \multicolumn{5}{|l|}{ GAD } \\
\hline Group & $0.31(0.15$ to 0.47$)$ & $0.001 * \star$ & 0.32 (0.16 to 0.48$)$ & $0.001^{* *}$ \\
\hline Baseline & $-0.03(-0.15$ to 0.09$)$ & 0.60 & $-0.01(-0.09$ to 0.07$)$ & 0.77 \\
\hline$G \times B$ & $0.04(-0.13$ to 0.20$)$ & 0.65 & & \\
\hline \multicolumn{5}{|l|}{ SAD } \\
\hline Group & 0.43 (0.29 to 0.57$)$ & $<0.001^{* * *}$ & $0.43(0.29$ to 0.56$)$ & $<0.001^{* * *}$ \\
\hline Baseline & $0.06(-0.04$ to 0.17$)$ & 0.21 & $0.04(-0.03$ to 0.11$)$ & 0.29 \\
\hline$G \times B$ & $-0.06(-0.20$ to 0.09$)$ & 0.43 & & \\
\hline \multicolumn{5}{|l|}{ OCD } \\
\hline Group & 0.35 (0.23 to 0.46$)$ & $<0.001^{* * *}$ & 0.35 (0.24 to 0.47$)$ & $<0.001^{* * *}$ \\
\hline Baseline & 0.09 (0.004 to 0.17$)$ & $0.041^{*}$ & 0.11 (0.05 to 0.17$)$ & $0.001 * *$ \\
\hline$G \times B$ & $0.04(-0.07$ to 0.16$)$ & 0.46 & & \\
\hline \multicolumn{5}{|l|}{ PTSD } \\
\hline Group & $0.20(-0.17$ to 0.56$)$ & 0.25 & $0.20(-0.16$ to 0.56$)$ & 0.24 \\
\hline Baseline & $-0.01(-0.28$ to 0.27$)$ & 0.96 & $0.07(-0.12$ to 0.26$)$ & 0.41 \\
\hline$G \times B$ & $0.16(-0.22$ to 0.53$)$ & 0.37 & & \\
\hline \multicolumn{5}{|c|}{ Panic disorder } \\
\hline Group & 0.14 (0.05 to 0.24$)$ & $0.006^{* *}$ & 0.14 (0.05 to 0.24$)$ & $0.005^{* *}$ \\
\hline Baseline & $-0.01(-0.11$ to 0.09$)$ & 0.85 & $-0.01(-0.08$ to 0.06$)$ & 0.81 \\
\hline$G \times B$ & $0.002(-0.10$ to 0.10$)$ & 0.96 & & \\
\hline
\end{tabular}

and Fig. 2). Group membership was a significant predictor of the remission rate $(P<0.01)$ but baseline severity was not. Paralleling these results, inclusion of treatment group in the model reduced between-group heterogeneity for GAD ( $I^{2}$ decreasing from $83 \%$ to $67 \%)$, SAD ( $84 \%$ to $49 \%)$, OCD $(75 \%$ to $52 \%)$ and panic disorder $(86 \%$ to $77 \%)$, although it did not reduce heterogeneity for PTSD (85\% to $84 \%)$. Including the main effect of baseline reduced heterogeneity for OCD only ( $52 \%$ to $14 \%)$, whereas
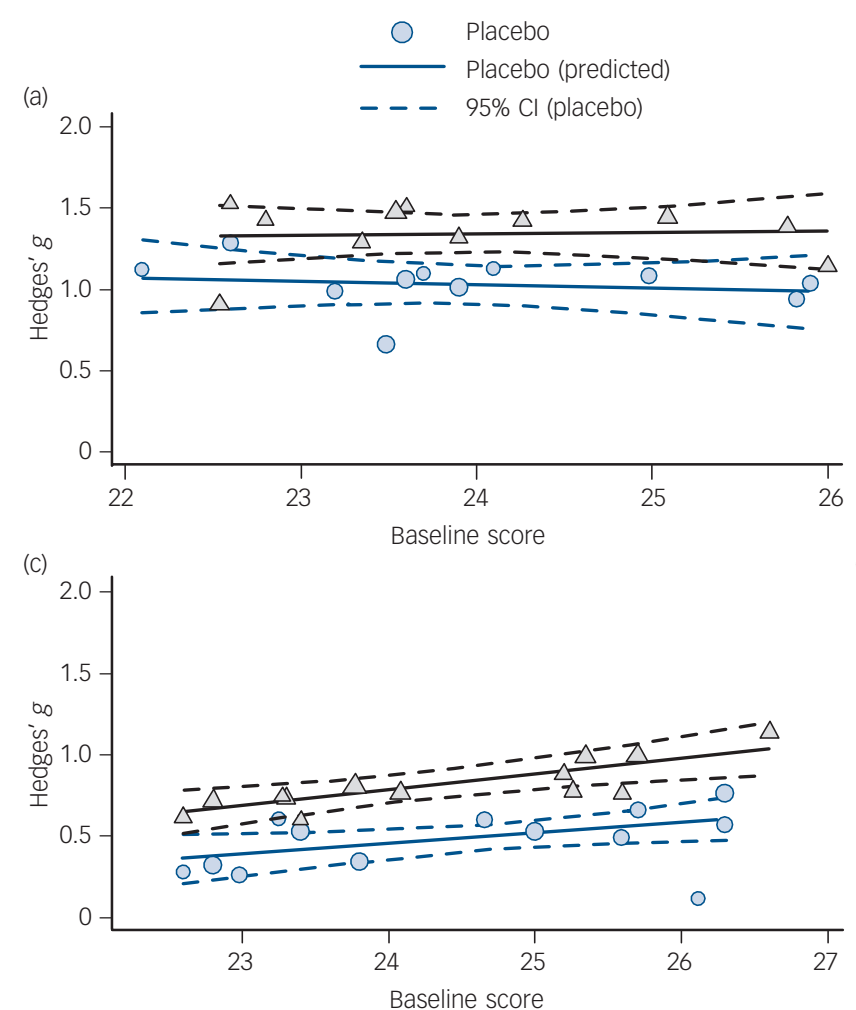

including the interaction did not reduce heterogeneity further for any disorder.

\section{Sensitivity analyses}

In our secondary analysis baseline severity was not a significant predictor of the drug-placebo difference for any disorder (see online Table DS4). The expanded set of trials included 9
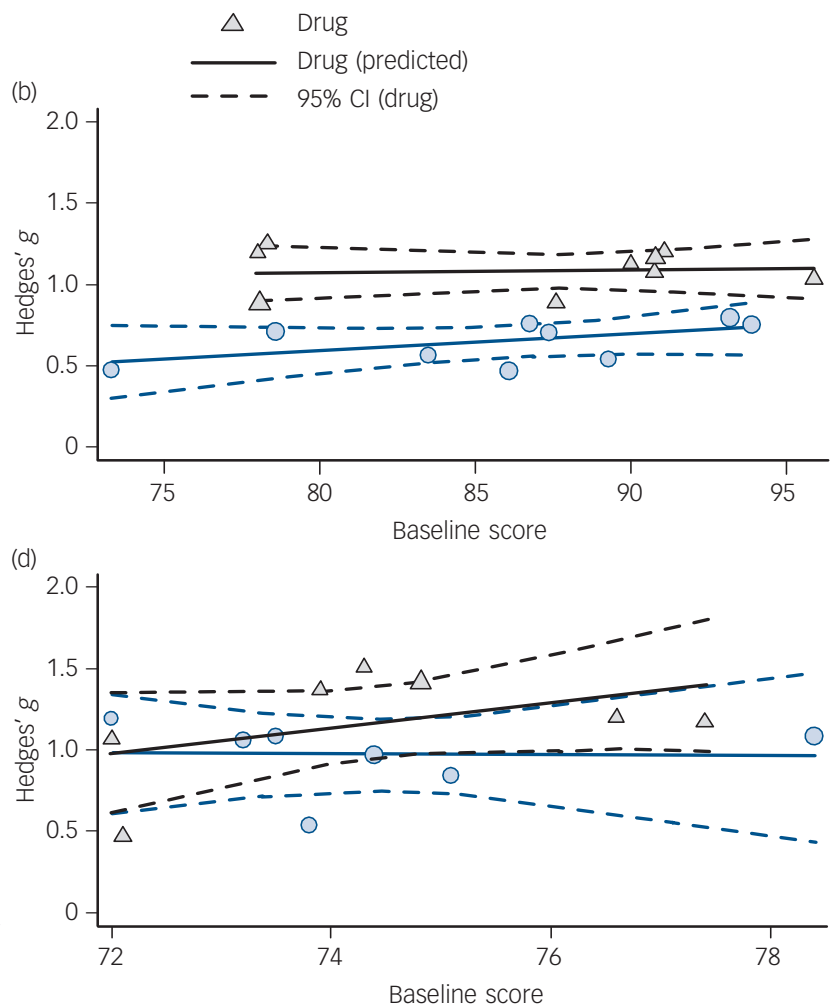

Fig. 1 Meta-regression analysis for (a) generalised anxiety disorder, (b) social anxiety disorder, (c) obsessive-compulsive disorder and (d) post-traumatic stress disorder. Data points are sized in proportion to the inverse of their standard error. 


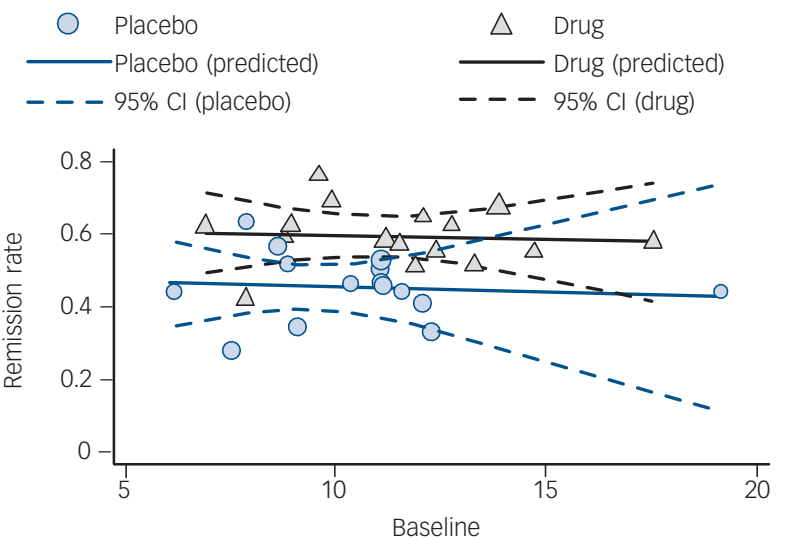

Fig. 2 Meta-regression analysis for panic disorder. Data points are sized in proportion to the inverse of their standard error.

additional trials for GAD, 10 additional trials for SAD, 6 additional trials for OCD, 6 additional trials for PTSD and 6 additional trials for panic disorder (see online data supplement for references). The range of baseline severity was increased slightly to substantially for all disorders in this expanded set of trials. However, no statistically significant interaction effect was found for any disorder, although the main effect of baseline became statistically significant for GAD, PTSD and SAD, in addition to OCD (see online Table DS5).

\section{Discussion}

We found no evidence that baseline severity of disorder affected the efficacy of second-generation antidepressants in the shortterm treatment of anxiety disorders. This finding stands in remarkable contrast to the reports of studies investigating major depressive disorder. For OCD, baseline severity did predict change in score in both placebo and drug groups but no differential effect was apparent. This suggests that patients with more severe OCD may show substantially greater improvement with antidepressant treatment than patients with milder OCD, but this is not due to improved antidepressant efficacy in severe disorder. Our sensitivity analysis including 37 additional trials suggested that a similar regression to the mean effect might also occur in other anxiety disorders, but confirmed the lack of evidence for an interaction effect in all anxiety disorders.

Effect sizes for the drug-placebo difference were unaffected by inclusion of baseline severity as a main effect or in interaction with group, but they were generally smaller than a criterion for clinical significance previously used (although without clear justification) for major depression $(d=0.5) .{ }^{12}$ However, it has been shown that effect sizes exceeding 0.5 are not achieved by most current treatments, either in psychiatry or in general medicine. ${ }^{30}$ Furthermore, clinical significance is both context- and disorder-specific. ${ }^{31}$ An empirically derived criterion of $d=0.24$ has been proposed as a more meaningful threshold for clinically significant efficacy in major depression. ${ }^{32}$ Although not all included anxiety disorders met that threshold in our primary analysis, the drug-placebo difference may have been underestimated slightly owing to our analytical approach. We have previously analysed the trials included in this study by conventional meta-analytic methods and found effect sizes of 0.27 and greater. ${ }^{23}$ Further research is required to establish whether antidepressant efficacy in the different anxiety disorders may be considered clinically significant in all or in subsets of patients. Additionally, the expected efficacy of medication is only one of many factors that play a part in the decision to prescribe antidepressants for an individual patient. Other issues - such as the expected burden of side-effects, the burden posed by the disorder itself, the anticipated course of the disorder, and the availability, acceptability and efficacy of alternative treatment options such as psychotherapy - must also be considered. ${ }^{33}$ These considerations might lead to different prescribing decisions for patients with mild $v$. severe anxiety disorders, even in the absence of differential efficacy.

\section{Comparison with depression}

For major depressive disorder it has been suggested that the threshold for 'antidepressant-treatable' depression should be higher, ${ }^{34}$ given that patients with mild depression do not show a strong differential response to antidepressants over placebo. It is possible that this threshold needs to be even higher in anxiety disorders, and that we would find greater efficacy of antidepressants in patients with more severe anxiety than we do in patients with less extreme anxiety. Another possible explanation lies in the difference in chronicity between anxiety disorders and major depression. Depressive episodes tend to be relatively short, with a median duration of 3-6 months, ${ }^{3,35}$ whereas the median duration of an anxiety episode has been estimated at 16 months. ${ }^{3}$ However, duration of a depressive episode is positively correlated with severity. ${ }^{3}$ This suggests that (all other things being equal) people with mild depression are more likely to achieve spontaneous remission within the short time frame of a clinical trial than people with severe depression. Since a drug effect cannot be demonstrated in spontaneous remission, efficacy would be expected to be reduced in a patient group where this is likely to occur. Consequently, the correlation between severity and episode duration may explain the increase in antidepressant efficacy with increasing depression severity. Although severity also correlates with episode duration in anxiety disorders, ${ }^{36}$ this may play less of a part within the context of a short clinical trial. Given the long median duration of an episode of anxiety, spontaneous remission rates would be expected to be relatively low across the severity range.

We may also have been unable to detect a relationship between baseline severity and antidepressant efficacy owing to a restriction in range. As trials generally have a minimum severity requirement, as well as exclusion criteria that tend to exclude severely ill patients (e.g. regarding treatments received and comorbidity), baseline severity was restricted to the moderate to very severe range in our primary analyses, although the range was extended somewhat in our sensitivity analyses. However, previous studies in major depression which were able to detect a relationship between baseline severity and antidepressant efficacy had similarly restricted severity ranges. We may also have had insufficient power to find a statistically significant interaction: although the total number of included trials was large, the number of trials per disorder was limited to 7-16. However, the results do not suggest a meaningful trend towards increasing efficacy with increasing severity, except possibly in PTSD, for which we had the fewest available trials. The estimates of the interaction effect were substantially lower $(\leqslant 0.04$, except for PTSD) or even in the opposite direction as the estimate previously reported for depression. ${ }^{12}$ These estimates are small enough to be of limited clinical relevance, although some confidence intervals encompass values that might be considered clinically relevant. Furthermore, our secondary analyses, which included six to ten additional trials per disorder, also showed no evidence of increasing efficacy with increasing severity, even for PTSD. Larger samples would be required to conclusively exclude the possibility of even small 
interaction effects, but unfortunately the number of randomised trials that have been conducted is limited, and consequently such samples are not available.

\section{Strengths and limitations}

Among the strengths of the study is the fact that we were able to obtain a data-set that was free from the influence of publication or outcome reporting bias. An additional strength is the high quality of the included trials. As these trials were conducted for the purpose of obtaining marketing approval, they were required to meet strict standards on internal validity (masking, randomisation, etc.). This also ensured that trials conducted for the treatment of the same disorder were in general comparable. A limitation is that we were forced to use a different outcome measure for panic disorder (remission) from that used for the other disorders; consequently we cannot rule out the possibility that an interaction might have been found if we had used a continuous outcome (such as change) instead. An additional, important limitation is that we did not have access to individual patient data and hence used summary data instead. Use of summary data necessarily causes a loss of information, due to averaging out inter-individual variation within trials, and a concomitant loss of power. Although individual patient data have historically been difficult to obtain, novel initiatives such as the Yale University Open Data Access Project (http://yoda.yale.edu) and Clinical Study Data Request (https://clinicalstudydatarequest.com) are now beginning to increase the accessibility of such data to researchers. Given our current results, which suggest that the influence of baseline severity on antidepressant efficacy may be different for anxiety disorders compared with depression, future research making use of individual patient data is essential to provide a definitive answer to this important question.

\section{Clinical implications}

It has been recommended that treatment with antidepressants should be withheld for mild depression. ${ }^{34,37}$ The absence of an interaction between treatment group and baseline severity in predicting symptom change in our study shows that this advice cannot be simply extrapolated to anxiety disorders, and it would therefore be premature to recommend that antidepressants be withheld for mild anxiety. What defines a clinically relevant effect size remains a matter of debate, but if the effect of antidepressants on anxiety is considered clinically relevant, these results suggest that antidepressants may be prescribed to patients with anxiety regardless of symptom severity.

Ymkje Anna de Vries, MSC, Peter de Jonge, PhD, Interdisciplinary Center Psychopathology and Emotion Regulation, Department of Psychiatry, University Medical Center Groningen, University of Groningen, The Netherlands; Edwin van den Heuvel, PhD. Department of Mathematics and Computer Science, Technica University Eind oven, Eindhoven, The Netherlands; Erick H. Turner, MD, Behaviol Health and Neurosciences Division, Portland Veterans Affairs Medical Center, and Health and Neurosciences Division, Portland Veterans Affairs Medical Center, and
Department of Psychiatry, Oregon Health and Science University, Portland, Oregon, USA; Annelieke M. Roest, PhD, Interdisciplinary Center Psychopathology and Emotion Regulation, Department of Psychiatry, University Medical Center Groningen University of Groningen, The Netherlands

Correspondence: Ymkje Anna de Vries, Department of Psychiatry, University Medical Center Groningen, Hanzeplein 1, 9713 GZ Groningen, The Netherlands. Email: y.a.de.vries@umcg.n!

First received 31 Mar 2015, final revision 7 Aug 2015, accepted 21 Sep 2015

\section{Funding}

The study was supported by grant KS2011(1)-120 from the Dutch Brain Foundation to P.J. The funder had no role in the study design, data collection and analysis, decision to publish or preparation of the manuscript.

\section{References}

1 Kessler RC, Chiu WT, Demler O, Walters EE. Prevalence, severity, and comorbidity of 12-month DSM-IV disorders in the National Comorbidity Survey Replication. Arch Gen Psychiatry 2005; 62: 617-27.

2 Kessler RC, Berglund PA, Demler O, Jin R, Merikangas KR, Walters EE. Lifetime prevalence and age-of-onset distributions of DSM-IV disorders in the National Comorbidity Survey Replication. Arch Gen Psychiatry 2005; 62: 593-603.

3 Penninx BWJH, Nolen WA, Lamers F, Zitman FG, Smit JH, Spinhoven P, et al. Two-year course of depressive and anxiety disorders: results from the Netherlands Study of Depression and Anxiety (NESDA). J Affect Disord 2011; 133: $76-85$.

4 Bruce SE, Yonkers KA, Otto MW, Eisen JL, Weisberg RB, Pagano M, et al. Influence of psychiatric comorbidity on recovery and recurrence in generalized anxiety disorder, social phobia, and panic disorder: a 12-year prospective study. Am J Psychiatry 2005; 162: 1179-87.

5 Whiteford HA, Degenhardt L, Rehm J, Baxter AJ, Ferrari AJ, Erskine HE, et al. Global burden of disease attributable to mental and substance use disorders: findings from the Global Burden of Disease Study 2010. Lancet 2013; 382: 1575-86.

6 Hidalgo RB, Tupler LA, Davidson JRT. An effect-size analysis of pharmacologic treatments for generalized anxiety disorder. J Psychopharmacol 2007; 21: 864-72.

7 Hansen RA, Gaynes BN, Gartlehner G, Moore CG, Tiwari R, Lohr KN. Efficacy and tolerability of second-generation antidepressants in social anxiety disorder. Int Clin Psychopharmacol 2008; 23: 170-9.

8 Soomro GM, Altman DG, Rajagopal S, Oakley Browne M. Selective serotonin re-uptake inhibitors (SSRIS) versus placebo for obsessive compulsive disorder (OCD). Cochrane Database Syst Rev 2008; 1: CD001765.

9 Stein DJ, Ipser JC, Seedat S. Pharmacotherapy for post traumatic stress disorder (PTSD). Cochrane Database Syst Rev 2006; 1: CD002795.

10 Andrisano C, Chiesa A, Serretti A. Newer antidepressants and panic disorder: a meta-analysis. Int Clin Psychopharmacol 2013; 28: 33-45.

11 Khan A, Leventhal RM, Khan SRF, Brown WA. Severity of depression and response to antidepressants and placebo: an analysis of the Food and Drug Administration database. J Clin Psychopharmacol 2002; 22: 40-5.

12 Kirsch I, Deacon BJ, Huedo-Medina TB, Scoboria A, Moore TJ, Johnson BT. Initial severity and antidepressant benefits: a meta-analysis of data submitted to the Food and Drug Administration. PLOS Med 2008; 5: e45.

13 Barbui C, Cipriani A, Patel V, Ayuso-Mateos JL, van Ommeren M. Efficacy of antidepressants and benzodiazepines in minor depression: systematic review and meta-analysis. Br J Psychiatry 2011; 198: 11-6.

14 Khan A, Bhat A, Faucett J, Kolts RL, Brown WA. Antidepressant-placebo differences in 16 clinical trials over 10 years at a single site: role of baseline severity. Psychopharmacology (Berl) 2011; 214: 961-5.

15 Khan A, Brodhead AE, Kolts RL, Brown WA. Severity of depressive symptoms and response to antidepressants and placebo in antidepressant trials. J Psychiatr Res 2005; 39: 145-50.

16 Fournier JC, Derubeis RJ, Hollon SD, Dimidjian S, Amsterdam JD, Shelton RC, et al. Antidepressant drug effects and depression severity. JAMA 2010; 303: 47-53.

17 Gibbons RD, Hur K, Brown CH, Davis JM, Mann JJ. Benefits from antidepressants: synthesis of 6-week patient-level outcomes from double-blind placebo-controlled randomized trials of fluoxetine and venlafaxine. Arch Gen Psychiatry 2012; 69: 572-9.

18 Ackerman DL, Greenland S. Multivariate meta-analysis of controlled drug studies for obsessive-compulsive disorder. J Clin Psychopharmacol 2002; 22 309-17.

19 Sugarman MA, Loree AM, Baltes BB, Grekin ER, Kirsch I. The efficacy of paroxetine and placebo in treating anxiety and depression: a meta-analysis of change on the Hamilton Rating Scales. PLOS One 2014; 9: e106337.

20 Stein DJ, Stein MB, Pitts CD. Predictors of response to pharmacotherapy in social anxiety disorder: an analysis of 3 placebo-controlled paroxetine trials. J Clin Psychiatry 2002; 63: 152-5.

21 Montgomery SA. Implications of the severity of social phobia. J Affect Disord 1998; 50 (suppl 1): s17-22.

22 Pollack MH, Meoni P, Otto MW, Hackett D. Predictors of outcome following venlafaxine extended-release treatment of DSM-IV generalized anxiety disorder: a pooled analysis of short-and long-term studies. J Clin Psychopharmacol 2003; 23: 250-9.

23 Roest AM, de Jonge P, Williams CD, de Vries YA, Schoevers RA, Turner EH. Reporting bias in clinical trials investigating the efficacy of second-generation antidepressants in the treatment of anxiety disorders: a report of 2 metaanalyses. JAMA Psychiatry 2015; 72: 500-10. 
24 Turner EH. How to access and process FDA drug approval packages for use in research. BMJ 2013; 347: f5992.

25 Morris SB, DeShon RP. Combining effect size estimates in meta-analysis with repeated measures and independent-groups designs. Psychol Methods 2002 7: $105-25$

26 Adli M, Baethge C, Heinz A, Langlitz N, Bauer M. Is dose escalation of antidepressants a rational strategy after a medium-dose treatment has failed? A systematic review. Eur Arch Psychiatry Clin Neurosci 2005; 255 387-400.

27 Ioannidis JPA, Patsopoulos NA, Evangelou E. Uncertainty in heterogeneity estimates in meta-analyses. BMJ 2007; 335: 914-6.

28 Bandelow B, Reitt M, Rover C, Michaelis S, Gorlich Y, Wedekind D. Efficacy of treatments for anxiety disorders: a meta-analysis. Int Clin Psychopharmacol 2015; 30: 183-92.

29 Hoskins M, Pearce J, Bethell A, Dankova L, Barbui C, Tol WA, et al. Pharmacotherapy for post-traumatic stress disorder: systematic review and meta-analysis. Br J Psychiatry 2015; 206: 93-100.

30 Leucht S, Hierl S, Kissling W, Dold M, Davis JM. Putting the efficacy of psychiatric and general medicine medication into perspective: review of meta-analyses. Br J Psychiatry 2012; 200: 97-106.
31 Kraemer HC, Kupfer DJ. Size of treatment effects and their importance to clinical research and practice. Biol Psychiatry 2006; 59: 990-6.

32 Cuijpers $P$, Turner EH, Koole SL, van Dijke A, Smit F. What is the threshold for a clinically relevant effect? The case of major depressive disorders. Depress Anxiety 2014; 31: 374-8

33 National Institute for Health and Care Excellence. Common Mental Health Disorders: Identification and Pathways to Care (Clinical Guideline CG123). NICE, 2011.

34 Baumeister $\mathrm{H}$. Inappropriate prescriptions of antidepressant drugs in patients with subthreshold to mild depression: time for the evidence to become practice. J Affect Disord 2012; 139: 240-3.

35 Spijker J, de Graaf R, Bijl RV, Beekman ATF, Ormel J, Nolen WA. Duration of major depressive episodes in the general population: results from The Netherlands Mental Health Survey and Incidence Study (NEMESIS). Br J Psychiatry 2002; 181: 208-13.

36 Hendriks SM, Spijker J, Licht CMM, Beekman ATF, Penninx BWJH. Two-year course of anxiety disorders: different across disorders or dimensions? Acta Psychiatr Scand 2013; 128: 212-21.

37 National Institute for Health and Care Excellence. Depression in Adults: Recognition and Management (Clinical Guideline CG90). NICE, 2009. poems

by doctors

\section{A Technique for Operating on the Past}

\section{Maya Catherine Popa}

My great-grandfather held a brain and studied it for signs of music Like all the men in my family, he was a close reader and musician.

The day the KGB arrived to take him, his students misplaced the combination to the ether closet. I see him in the armamentarium

choosing between scalpels and scopes, escaping across

the Carpathians in peasant's clothes. True, he did not love the state,

a symphony full of poorly written solos. But he could hold a brain

more steady than any in the University, interrogate its perilous

Iongitudes, cardinal directions for taste and melancholy, yellow tulips, joy. I see him peeling back the hair, that quiet, necessary artifice,

to reveal a nesting doll of impulses, then reciting the cold, hard rain of these connections at conferences in Cambridge. A halo of stage

whispers as he came as close to candor with the mind as was possible. He understood the officer's parietal lobe where his punishment

waited to be articulated, its obstinacy illustrated in early phrenology by the silhouette of a ram. Always the doctor's burden to reason

with that which cannot easily be reasoned. I see him make the first incision, certain, gentle as a breaststroke in the Black Sea. He knew

how to tell the brain a story, listen when told one in return. Knew that engine of ephemera could be a sentencing, a silence or a song.

From The Hippocrates Prize 2015: The Winning and Commended Poems, selected by T Dalrymple, R Gross, F Oyebode and S Rae, eds MW Hulse \& DRJ Singer. The Hippocrates Press, 2015. (c) Maya Catherine Popa. Reprinted with permission. 